\title{
Reduction of Ischemic and Oxidative Damage to the Hypothalamus by Hyperbaric Oxygen in Heatstroke Mice
}

\author{
Po-An Tai, ${ }^{1,2}$ Chen-Kuei Chang, ${ }^{3,4}$ Ko-Chi Niu, ${ }^{5}$ Mao-Tsun Lin, ${ }^{3,5}$ Wen-Ta Chiu, ${ }^{3}$ \\ and Jia-Wei Lin ${ }^{1,6}$ \\ ${ }^{1}$ Graduate Institute of Clinical Medicine, Taipei Medical University, Taipei 110, Taiwan \\ ${ }^{2}$ Department of Surgery, Buddhist Tzu Chi General Hospital, Taipei 231, Taiwan \\ ${ }^{3}$ Graduate Institute of Disease Pvention and Control, Taipei Medical University, Taipei 110, Taiwan \\ ${ }^{4}$ Department of Neurosurgery, Mackay Memorial Hospital, Taipei 104, Taiwan \\ ${ }^{5}$ Department of Medical Research, Chi Mei Medical Center, Tainan 710, Taiwan \\ ${ }^{6}$ Department of Neurosurgery, Shuang Ho Hospital, Taipei Medical University, Taipei 110, Taiwan
}

Correspondence should be addressed to Jia-Wei Lin, ns246@tmu.edu.tw

Received 4 March 2010; Accepted 5 May 2010

Academic Editor: Thomas Griffith

Copyright ( $) 2010$ Po-An Tai et al. This is an open access article distributed under the Creative Commons Attribution License, which permits unrestricted use, distribution, and reproduction in any medium, provided the original work is properly cited.

\begin{abstract}
The aims of the present paper were to ascertain whether the heat-induced ischemia and oxidative damage to the hypothalamus and lethality in mice could be ameliorated by hyperbaric oxygen therapy. When normobaric air-treated mice underwent heat treatment, the fractional survival and core temperature at 4 hours after heat stress were found to be 0 of 12 and $34^{\circ} \mathrm{C} \pm 0.3^{\circ} \mathrm{C}$, respectively. In hyperbaric oxygen-treated mice, when exposed to the same treatment, both fractional survival and core temperature values were significantly increased to new values of $12 / 12$ and $37.3^{\circ} \mathrm{C} \pm 0.3^{\circ} \mathrm{C}$, respectively. Compared to normobaric air-treated heatstroke mice, hyperbaric oxygen-treated mice displayed lower hypothalamic values of cellular ischemia and damage markers, prooxidant enzymes, proinflammatory cytokines, inducible nitric oxide synthase-dependent nitric oxide, and neuronal damage score. The data indicate that hyperbaric oxygen may improve outcomes of heatstroke by normalization of hypothalamic and thermoregulatory function in mice.
\end{abstract}

\section{Introduction}

Hyperbaric oxygen $(\mathrm{HBO})$ therapy is a noninvasive medical strategy in which a person breathes $100 \%$ oxygen at a pressure greater than normal [1]. We have previously demonstrated that $\mathrm{HBO}$ therapy may resuscitate anesthetized rats that had a heatstroke by reducing multiple organ dysfunction or failure [2-4]. A heatstroke patient with multiple organ dysfunction has also been successfully treated with $\mathrm{HBO}$ [5]. Although, $\mathrm{HBO}$ is beneficial in treating heatstroke, however, the mechanisms underlying the beneficial effects of $\mathrm{HBO}$ in heatstroke remain unclear.

It is well-known that glutamate and lactate-to-pyruvate ratio are cellular ischemia markers, whereas glycerol is a cellular damage marker [6]. Heatstroke mice display increased production of glutamate, lactate-to-pyruvate ratio, and glycerol in hypothalamus [7, 8]. The thermoregulatory deficits (e.g., hypothermia occurred during room temperature exposure) that occurred after heatstroke formation in mice [7-10] may have resulted from hypothalamic ischemia and neuronal damage. Because the hypothalamus regulates body temperature [11], it is possible that thermoregulatory deficits are induced during heatstroke. Multiple organ dysfunction or failure that occurred during heatstroke may be related to alteration of hypothalamic-pituitary-adrenalaxis-mechanisms $[12,13]$. It is not known whether the heat-induced hypothalamic dysfunction and mortality in mice can be ameliorated by $\mathrm{HBO}$ therapy.

To deal with the question, the aim of the present study was attempted to assess the effects of $\mathrm{HBO}$ on the heat-induced hypothermia and lethality in unanesthetized, unrestrained mice. In addition, the temporal profiles of cellular ischemia and oxidative damage markers as well as inflammatory cytokines in the hypothalamus were assessed in heatstroke mice with or without $\mathrm{HBO}$ therapy. 


\section{Materials and Methods}

2.1. Animals. All the experiments were performed in accordance with the ethical guidelines laid down by the committee for the purpose of control and supervision of experiments on animals of Chi Mei Medical Center (Tainan, Taiwan). Institute of Cancer Research (ICR) inbred male mice were given food and water ad libitum and acclimatized to room temperature at $24^{\circ} \mathrm{C}$, relative humidity of $50 \pm 8 \%$, and a 12-hour dark/light cycle for 1 week before starting the experiment. These ICR strain mice were purchased from National Taiwan University (Taipei, Taiwan, ROC).

2.2. The Mouse Model of Heatstroke. Institute of Cancer Research inbred male mice, aged 8 to 10 weeks and weighing 23 to $25 \mathrm{~g}$, were exposed to heat stress treatment $\left(42.4^{\circ} \mathrm{C}\right.$; relative humidity, $50 \%-55 \%$; 1 hour) in an environmentcontrolled chamber. The time at which mice were removed from the environmental chamber was called 0 hours. The heat-stressed mice were returned to normal room temperature $\left(24^{\circ} \mathrm{C}\right)$ at the end of the heat treatment. Mice that survived on day 4 of heat treatment were considered survivors, and the data were used for analysis of the results. Core temperatures were measured every 5 minutes with a copper constantan thermocouple inspected into the rectum and connected to a thermometer (HR1300; Yokogawa, Tokyo, Japan). After the 1-hour heating period, animals were fed properly and hydrated. Heatstroke resembles sepsis in many aspects. Similar to many sepsis studies, in this study, we used death as an end point in conscious mice. The murine model of heatstroke has been detailed previously by several investigators [7-10]. As demonstrated in our previous study $[7,8]$, all heat-stressed mice survived 4 hours after whole body heating (WBH). Therefore, in the present study, physiologic parameter measurements and histologic verification were performed at 4 hours after heat treatment.

2.3. Hyperbaric Oxygen Therapy. Three groups of animals were designated for the experiment. In the normothermic (NT) groups, their core temperatures were found to be $37.1^{\circ} \mathrm{C}$ to $37.5^{\circ} \mathrm{C}$ at a room temperature of $24^{\circ} \mathrm{C}$ as well as a room oxygen content in air of $21 \%$. The $\mathrm{PO} 2$ of inspired oxygen (partial pressure of oxygen $=20 \mathrm{kpa}$ ) was calculated by multiplying $101 \mathrm{kPa}$ by $21 \%$. In the hyperbaric oxygen (HBO)-treated heatstroke group, mice were resuscitated directly after instrumentation with $100 \%$ oxygen at $253 \mathrm{kpa}$ (oxygen content in hyperbaric oxygen air) for 2 hours. The chamber filled with pure oxygen $(100 \%)$ was pressurized to $253 \mathrm{kpa}$ at a rate of $51 \mathrm{kpa} / \mathrm{min}$ for 2 hours and was terminated at the decompression rate of $20 \mathrm{kpa} / \mathrm{min}$. In the untreated heatstroke group, mice were exposed to normobaric air (NBA) $(21 \% ; 101 \mathrm{kpa})$.

\subsection{Experimental Groups}

Experiment 1 (effect of heat on percent survival and core temperature). A dose of NBA or $\mathrm{HBO}(n=12$ for each group) was randomly administered to mice 1 hour post-WBH, and its effects on percent survival and core temperature were assessed 4 days post-WBH and 4 hours post-WBH, respectively. Heatstroke mice were exposed to $42.4^{\circ} \mathrm{C}$ for 1 hour and then allowed to recover at room temperature $\left(24^{\circ} \mathrm{C}\right)$. Core temperatures were measured at 4 hours post-WBH and percent survivals were counted on 4 days post-WBH.

Experiment 2 (effect of heat on hypothalamic neuronal damage score). A dose of NBA or HBO ( $n=8$ for each group) was randomly administered to mice 1 hour post$\mathrm{WBH}$, and its effects on neuronal damage score in the hypothalamus were assessed 4 hours post-WBH.

Experiment 3 (effect of heat on hypothalamic levels of ischemia and neuronal damage markers). a dose of NBA or $\mathrm{HBO}$ ( $n=8$ for each group) was randomly administered to mice 1 hour post-WBH, and its effects on nitric oxide (NO), dihydroxybenzoic acid (DHBA), and cellular ischemia (eg., glutamate and lactate-to-pyruvate ratio) and damage (eg., glycerol) markers of the hypothalamus were assessed 4 hours post-WBH.

Experiment 4 (effect of heat on hypothalamic levels of cytokines). A dose of NBA or HBO was randomly administered to mice 1 hour post-WBH, and its effects on hypothalamic levels of tumor necrosis factor- $\alpha$ (TNF- $\alpha$ ), interleukin- $1 \beta$ (IL-1 $\beta$ ), IL-10, and myeloperoxidase (MPO) were assessed 4 hours post-WBH.

Experiment 5 (effect of heat on hypothalamic levels of pro-oxidant enzymes (eg, malondialdehyde, GSSG) and anti-oxidant enzymes (eg, GSH, glutathione peroxidase, glutathione reductase)). a dose of NBA was randomly administered to mice 1 hour post-WBH, and its effects on hypothalamic levels of these pro-oxidant and anti-oxidant enzymes were assessed 4 hours post-WBH.

2.5. Histological Verification. At the end of 4 hour post$\mathrm{WBH}$, animals were sacrificed and their brains were removed, fixed in 10\% neutral buffered formalin, and embedded in paraffin blocks. Serial $(10 \mu \mathrm{m})$ sections through the hypothalamus were stained with hematoxylin and eosin for microscopic examination. The extent of hypothalamic neuronal damage was scored on a scale of $0-3$, modified from the grading system of Pulsinelli et al. [14], in which 0 is normal, 1 means that $\sim 30 \%$ of the neurons are damage, 2 means that $\sim 60 \%$ of the neurons are damaged, and 3 means that $100 \%$ of neurons are damaged. Each hemisphere was evaluated independently without knowledge of the experimental conditions.

2.6. Determination of Stable Nitric Oxide Metabolites (NOx). Samples of extracellular fluids of hypothalamus were collected, immediately separated, and stored at $-80^{\circ} \mathrm{C}$ until they could be assayed. The NOx concentrations in the hypothalamic dialysates were measured with the Eicom ENO-20 $\mathrm{NO}_{x}{ }^{-}$analysis system (Eicom, Kyoto, Japan) [15]. In the Eicom ENO-20 $\mathrm{NO}_{x}{ }^{-}$analysis system, after the $\mathrm{NO}_{2}{ }^{-}$ and $\mathrm{NO}_{3}{ }^{-}$in the sample have been separated by the column, 
the $\mathrm{NO}_{2}{ }^{-}$reacts in the acidic solution with the primary aromatic amine to produce an azo compound. After this, the addition of aromatic amines to the azo compound results in a coupling that produces a diazo compound, and the absorbance rate of the red color of this compound is then measured. The dialysates were injected onto a CMA600 microdialysis analyzer (Carnegie Medicine, Stockholm, Sweden) for measuring lactate, pyruvate, glycerol, and glutamate.

2.7. Measurement of Cytokines and DHBA. Samples of extracellular fluids of hypothalamus were collected, immediately separated, and stored at $-80^{\circ} \mathrm{C}$ until they could be assayed. We used commercially available ELISA kits for the determination of levels of TNF- $\alpha$, IL- $1 \beta$, or IL-10, according to the manufacture's instructions (Quantikine, R\&D System, MN, USA). The concentration of DHBA were measured by a modified procedure based on the hydroxylation of sodium salicylate by hydroxyl radicals, leading to the production of DHBA [16].

2.8. Biochemical Determination. Lipid peroxidation was assessed by measuring the levels of malondialdehyde (MDA) with 2-thiobarbituric acid (TBA) to form a chromospheres absorbing at $532 \mathrm{~nm}$ [17]. About $0.1 \mathrm{~g}$ of tissue was homogenized with $1.5 \mathrm{~mL}$ of $0.1 \mathrm{M}$ phosphate buffer at $\mathrm{pH} 3.5$. The reaction mixture $(0.2 \mathrm{~mL}$ of sample, $1.5 \mathrm{~mL}$ of $20 \%$ acetic acid, $0.2 \mathrm{~mL}$ of $8.1 \%$ sodium dodecyl sulfate, and $1.5 \mathrm{~mL}$ of aqueous solution of $0.8 \% \mathrm{TBA}$, up to $4 \mathrm{~mL}$ with distilled water) was heated to $95^{\circ} \mathrm{C}$ for 1 hour, and then $5 \mathrm{~mL}$ of N-butanol and pyridine $(15.1 \mathrm{vol} / \mathrm{vol})$ was added. The mixture was vortexed vigorously, centrifuged at $1500 \mathrm{~g}$ for 10 minutes, and the absorbance of the organic phose was measured at $532 \mathrm{~nm}$. The values were expressed as nanomoles of TBA-reactive substance (MDA equivalent) per milligram of protein.

Tissues were homogenized in 5\% 5-sulfosalicylic acid $(1: 10 \mathrm{wt} / \mathrm{vol})$ at $0^{\circ} \mathrm{C}$, and the supernatants were used for analysis of total and oxidized glutathione. Total glutathione [reduced-form glutathione (GSH) + oxidized-form glutathione (GSSG)] was analyzed according to the Tietze method [18] and GSSG was determined as described by Griffith [19]. The recycling assay for total glutathione is oxidized by 5,5-Dithiosis [2 acid] (DTNB) to give GSSG with stoichiometric formation of 5-thio-2-nitro-benzoic acid. GSSG is reduced to GSH by the action of the highly specific glutathione reductase (GR) and nicotinamide adenine dinucleotide phosphate (reduced form; NADPH). The rate of 5-thio-2-nitro-benzoic acid formation is followed at $412 \mathrm{~nm}$ and is proportional to the sum of GSH and GSSG present.

2.9. Determination of Inducible Nitric Oxide Synthase (inos)Positive Cells. Mice were sacrificed with overdose of an anesthetic and were transcardially perfused with heparinized 0.05-M phosphate-buffered saline (PBS), followed by icecold $15 \%$ sucrose in PBS. The brains were rapidly removed and frozen in liquid nitrogen. Coronal brain sections (5$\mu \mathrm{m}$ thick) were cut in a cryostat and were thaw-mounted on gelatin-coated slides. Sections were incubated with commercially available rabbit anti-NOS antiserum $(1: 50)$ diluted in $0.2 \%$ Triton X-100 $1 \%$ azide (Sigma, St. Louis, $\mathrm{MO} / \mathrm{PBS}$ at $4^{\circ} \mathrm{C}$ overnight then rinsed with $\mathrm{PBS}$ for 30 minutes and incubated in biotinylated goat anti rabbit immunoglobulin G $(1: 500)$ for 1 to 2 hours. The iNOSpositive cells were counted in each section through the hypothalamus.

2.10. Myelo-Peroxidase Activity. Myeloperoxidase (MPO) activity, an indicator of polymorphonuclear leukocyte accumulation, was determined in the hypothalamus as described previously [20] at 4 hours after heat stress. MPO activity was defined as the quantity of enzyme degrading $1 \mu \mathrm{mol}$ of peroxide of $\min ^{-1}$ at $37^{\circ} \mathrm{C}$ and was expressed in milliunits grams $^{-1}$ of wet tissue.

2.11. Statistics. Statistical significance of survival was assessed using a $\mathrm{X}^{2}$ method and followed by Fisher exact probability test. Core temperature, levels of cytokines, nitric oxide, glutamate, glycerol, dihydroxybenzoic acid, and lactate-to-pyruvate ratio were analyzed using the KruskalWallis $\mathrm{H}$ test, followed by several post hoc comparisons with Dunn method. The Wilcoxon's signed rank test was used to compare the neuronal damage across two groups. The Wilcoxon tests convert scores to ranks, a sum of the ranks is calculated, and critical values of the sum are provided to test the null hypothesis at a given significant level. The data were given as "median", first quartile, and third quartile. A $P$ value of less than .05 was considered as statistically significant.

\section{Results}

3.1. Physiologic Variables. As summarized in Table 1, percent survival value of NBA-treated heatstroke mice for 4 days post-WBH is $0 \%(n=12)$. The core temperature value of NBA-treated heatstroke mice for 4 hours post-WBH is $33.6 \pm 0.52^{\circ} \mathrm{C}(n=12$; Table 2$)$. When compared with those of NBA-treated heatstroke mice, HBO-treated heatstroke mice had higher values of both percent-survival $(100 \%, n=$ 12 ; Table 1$)$ and core temperature $\left(36.8^{\circ} \mathrm{C}, n=12\right.$; Table 2$)$. When the heat stress was terminated in the heatstroke group, they displayed body wet with salivary spreading and stagger.

3.2. Neuronal Damage Scores. As summarized in Table 3, the neuronal damage scores in the hypothalamus of NBAtreated heatstroke mice were significantly higher than those of NT mice. Photographs of neurodegenerative cells in the hypothalamus of NBA-treated heatstroke mouse were shown in Figure 1. In HBO-treated heatstroke mice, HBO therapy adopted 1-hour post-whole body heating (WBH) significantly reduced the increased neuronal damage scores in the hypothalamus obtained at 4 hours post-WBH (Table 3; Figure 1).

The values of neuronal damage scores in the hypothalamus of HBO-treated normothermic (NT) mice were indistinguishable from those of NT mice that received NBA (Table 3). 
TABLE 1: Mean $( \pm$ SE) of percent survival or fraction survival values for different groups of mice.

\begin{tabular}{lcc}
\hline Treatment groups & Survival, $\%$ & Fraction survival \\
\hline (1) Normothermic (NT) mice received normobaric air (NBA) & 100 & $12 / 12$ \\
(2) NT mice received hyperbaric oxygen (HBO) & 100 & $12 / 12$ \\
(3) Heatstroke (HS) mice received NBA & 0 & $0 / 12$ \\
(4) HS mice received HBO & 100 & $12 / 12 \quad P<.001^{*}$ \\
\hline
\end{tabular}

When exactly 4 days post-HS, the percent survival values were measured.

* Compared with group 1 .

${ }^{\dagger}$ Compared with group 3 .

TABle 2: Mean $( \pm$ SE) of core temperature values for different groups of mice ( $n=8$ per group).

\begin{tabular}{lc}
\hline Treatment groups & Core temperature, ${ }^{\circ} \mathrm{C}$ \\
\hline (1) Normothermic (NT) mice received normobaric air (NBA) & $37.1 \pm 0.07$ \\
(2) NT mice received hyperbaric oxygen (HBO) & $36.9 \pm 0.09$ \\
(3) Heatstroke (HS) mice received NBA & $33.6 \pm 0.22$ \\
(4) HS mice received HBO & $36.8 \pm 0.08$ \\
\hline
\end{tabular}

When exactly 4 hours post-HS, the core temperature values were measured.

* Compared with group 1.

${ }^{\dagger}$ Compared with group 3.

3.3. Ischemic and Oxidative Damage to the Hypothalamus. Figure 2 depicts the effects of heat exposure $\left(42.4^{\circ} \mathrm{C}\right.$ for 1 hour) on cellular levels of glutamate, glycerol, lactate-topyruvate ratio, nitrite, and DHBA in the hypothalamus in NT mice that received NBA, NT mice that received $\mathrm{HBO}$, NBA-treated heatstroke mice, and $\mathrm{HBO}$-treated heatstroke mice. In NBA-treated heatstroke groups, the cellular levels of glutamate, glycerol, lactate-to-pyruvate ratio, nitrite, and DHBA in the hypothalamus were all significantly higher at 4 hours after the termination of heat exposure than those of NTs. Resuscitation with HBO 1 hour post-WBH significantly attenuated the heat-induced increased levels of glutamate, glycerol, lactate-to-pyruvate ratio, nitrite, and DHBA in the hypothalamus. The basal levels of those biochemical parameters in NTs treated with $\mathrm{HBO}$ were indistinguishable from those of NTs that received HBO.

3.4. Overproduction of Proinflammatory Cytokines. As summarized in Figure 3, the values of hypothalamic TNF- $\alpha$, IL- $1 \beta$, and MPO activity of NBA-treated heatstroke mice obtained at 4 hours post-WBH were significantly higher than those of NCs. In HBO-treated heatstroke mice, $\mathrm{HBO}$ therapy adopted 1-hour post-WBH significantly suppressed the increased levels of hypothalamic TNF- $\alpha$, IL- $1 \beta$, and MPO activity obtained at 4 hours post-WBH. On the other hand, as compared to those of NBA-treated heatstroke mice, HBOtreated heatstroke mice had higher values of IL-10 in their hypothalamus obtained at 4 hours post-WBH.

3.5. Oxidative Damage to the Hypothalamus. As summarized in Figure 4, the values of pro-oxidant enzymes (eg, MDA and GSSG) and anti-oxidant enzymes (eg, GSH, GP, and GR) of NBA-treated heatstroke mice obtained at 4 hours post$\mathrm{WBH}$ were significantly higher and lower, respectively, than those of NTs. In HBO-treated mice, HBO therapy adopted 1-hour post-WBH significantly reversed the increased levels of both MDA and GSSG and decreased levels of GSH, GP, and GR in the hypothalamus. The basal levels of these parameters in the hypothalamus of HBO-treated NT mice were indistinguishable from those of NTs that received NBA (Figure 4).

3.6. Overexpression of $i$ NOS. As demonstrated in Table 4, the numbers of iNOS-positive cells in the hypothalamus of NBAtreated heatstroke mice obtained at 4 hours post-WBH were higher than those of NT groups. Photomicrographs of iNOSpositive cells in the hypothalamus of a NBA-treated HS mouse were shown in Figure 5. The heat-induced increased numbers of iNOS-positive cells were reduced significantly by HBO therapy (Table 4 and Figure 5).

\section{Discussion}

Our previous [7] and present results demonstrated that when exposed the unanesthetized, unrestrained mice to a hot environment $\left(42.4^{\circ} \mathrm{C}\right)$ for 1 hour, profound thermoregulatory deficit as well as mortality were observed. Our current results further showed that the heat-induced thermoregulatory deficit (evidenced by hypothermia occurrence during room temperature exposure) and mortality could be resulted from ischemia and oxidative damage to their hypothalamus (the essential thermoregulatory center in mammalian brain) in the mouse. Compared to normothermic mice, heatstroke mice had significantly higher levels of cellular ischemia (e.g., glutamate and lactate-to-pyruvate ratio) and damage (e.g., glycerol) markers in their hypothalamus [21-26]. These heatstroke mice also displayed significantly higher hypothalamic levels of reactive nitrogen species (e.g., iNOSdependent NO metabolites), reactive oxygen species (e.g., DHBA and superoxide anions), lipid peroxidation (e.g., MDA), and enzymatic pro-oxidants (e.g., GSSG/GSH), but lower hypothalamic levels of enzymatic anti-oxidant 


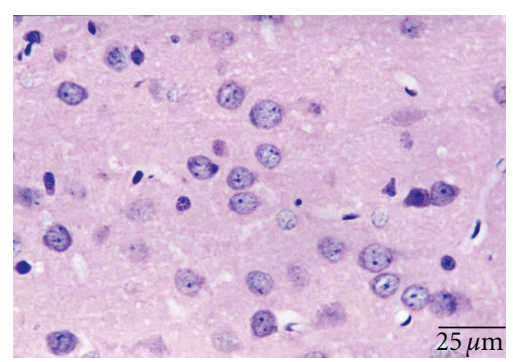

NT + NBA

(a)

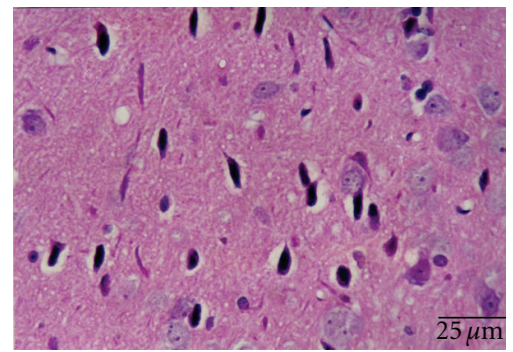

$\mathrm{HS}+\mathrm{NBA}$

(c)

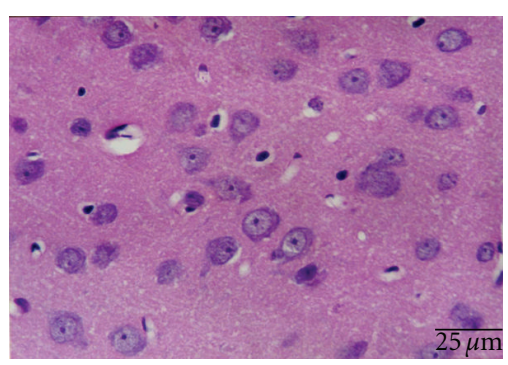

$\mathrm{NT}+\mathrm{HBO}$

(b)

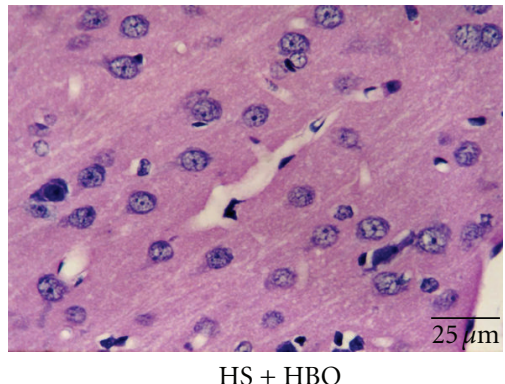

(d)

FIGURE 1: Photomicrographs of HE staining of the hypothalamus of normothermic (NT) mouse received normobaric air (NBA) (NT+NBA), NT mouse received hyperbaric oxygen $(\mathrm{HBO})(\mathrm{NT}+\mathrm{HBO})$, heatstroke $(\mathrm{HS})$ mouse received NBA $(\mathrm{HS}+\mathrm{NBA})$, and heatstroke mouse received $\mathrm{HBO}(\mathrm{HS}+\mathrm{HBO})$. The animals were killed at 4 hours after the termination of 1 -hour heat exposure or the equivalent time for the NT mouse.

TABLE 3: Hypothalamic neuronal damage score values for different groups of mice ( $n=8$ per group).

\begin{tabular}{lc}
\hline Treatment groups & $\begin{array}{c}\text { Neuronal damage scores } \\
\text { (Range, } 0-3)\end{array}$ \\
\hline (1) Normothermic (NT) mice received normobaric air (NBA) & $0(0,0)$ \\
(2) NT mice received hyperbaric oxygen (HBO) & $0(0,0)$ \\
(3) Heatstroke (HS) mice received NBA & $2(2,2)^{*}$ \\
(4) HS mice received HBO & $0(0,0)^{\dagger}$ \\
\hline
\end{tabular}

When exactly 4 hours post-HS, the neuronal damage scores were obtained.

${ }^{*} P<.01$ Compared with group 1 .

${ }^{\dagger} P<.01$ Compared with group 3 .

defences (e.g., activity of both GR and GPx). Increased production of reactive oxygen and nitrogen species has been reported to be directly involved in oxidative damage with cellular macromolecules in ischemic brain tissues, which lead to cell death [27].

Our previous experiments have demonstrated that HBO improves survival in anesthetized rats during heatstroke by reducing multiorgan dysfunction $[2,5]$. The present results further shows that $\mathrm{HBO}$ therapy effectively protects against heat-induced hypothalamic ischemia and oxidative damage, thermoregulatory dysfunction, and mortality in unanesthetized, unrestrained mice during heatstroke. The current findings are consistent with a previous study concerning with middle cerebral artery ligation-induced stroke [28]. They also observe that the outcomes of the conventional stroke in the rats can be improved by HBO. Conversely, the results are not supported by several investigations. For example, hyperbaric oxygen itself is able to induce acute cerebral toxicity in rats [29]. The cardiac arrest-induced neurologic deficit could be exacerbated by HBO therapy in dogs [30]. Increased lipid peroxidation and mortality in gerbilis after global ischemia could be exacerbated by HBO therapy [31].

In anesthetized rats, heat-induced hyperthermia, hypotension, and cerebral ischemia and damage occurred during heatstroke were associated with increased production of free radicals (specifically hydroxyl radicals and superoxide anions), higher lipid peroxidation, lower enzymatic antioxidant defenses, and higher pro-oxidants in the brain of heatstroke-effected rats [32]. Pretreatment with conventional hydroxyl radical scavengers (e.g., mannitol or $\alpha$-tocopherol) prevented the brain oxidative stress and increased subsequent survival time. The present results further demonstrated that in unanesthetized, unrestrained mice, HBO therapy was able to rescue mice from heat-induced unortality by reducing hypothalamic ischemia and oxidative damage. It should be mentioned that unanesthetized, unrestrained 

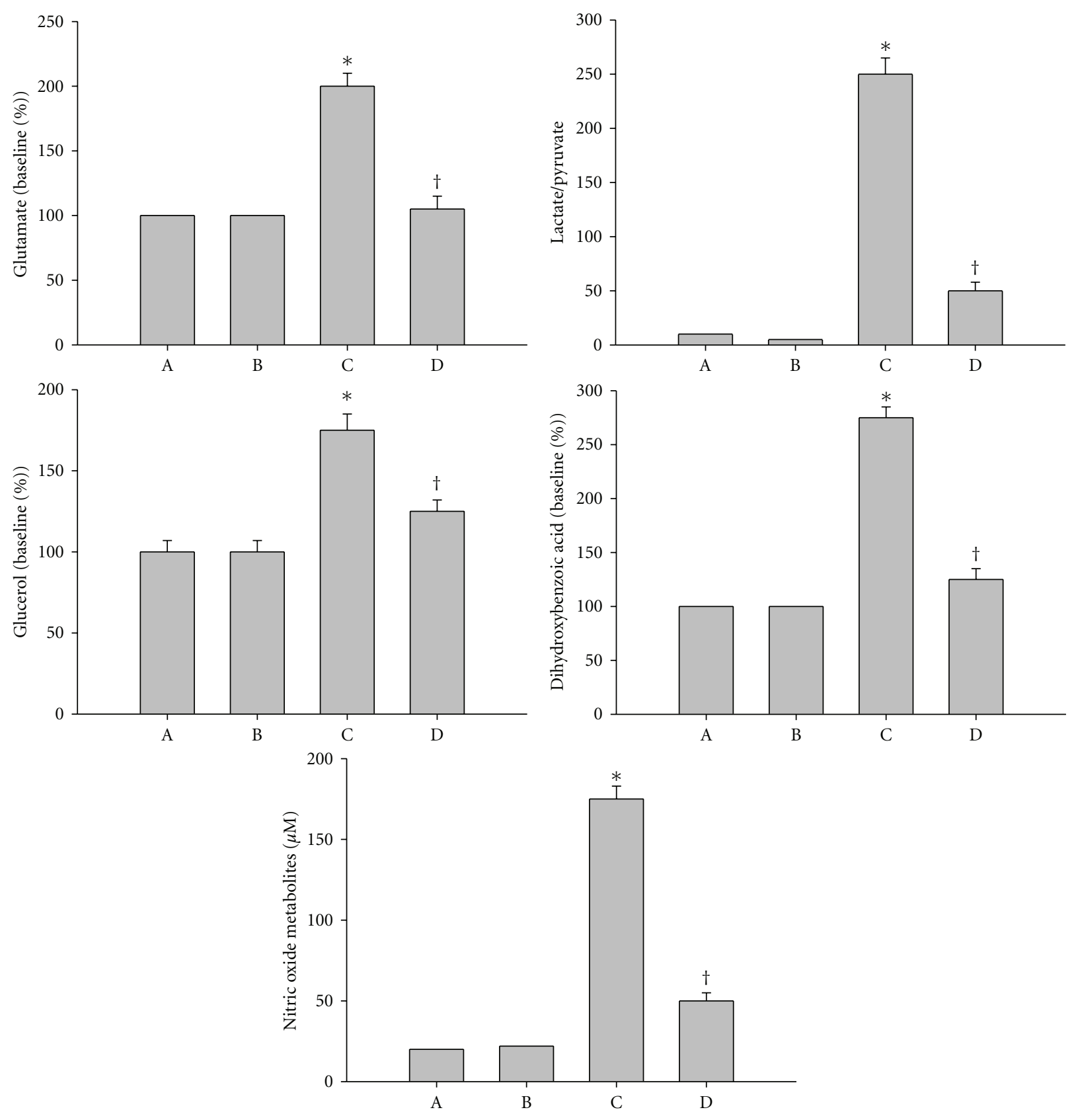

Figure 2: Effects of heat exposure $\left(42.4^{\circ} \mathrm{C}\right.$ for 1 hour) on extracellular levels of glutamate, lactate-to-pyruvate ratio, glycerol, dihydroxybenzoic acid, and nitric oxide in the hypothalamus of normothermic mice received normobaric air (A), normothermic mice received hyperbaric oxygen (B), heatstroke mice received normobaric air (C), and heatstroke mice received hyperbaric oxygen (D). Bars are mean \pm SE of eight mice per group. Samples were obtained at 4 hours after the termination of 1-hour heat exposure or the equivalent time for the normothermic controls. ${ }^{*} P<.01$ compared with corresponding control values in group (A). ${ }^{\dagger} P<.01$ compared with corresponding values in group $(\mathrm{C})$.

mice also displayed hyperthermia during heat stress but hypothermia 4 hours after heat stress. The heat-induced hypothermia may be due to hypothalamic dysfunction.

In the rodents, environmental heat stress increased cutaneous blood flow and metabolism and progressively decreased splanchnic blood flow. It was shown that ischemia and hypoxia led to elevation of nitric oxide level in the blood stream in anesthetized [32-34] or unanesthetized [35] rats.
Heat-induced multiple organ dysfunction could be greatly reduced by inducible or neuronal nitric oxide synthase inhibitors in anesthetized rat during heatstroke [33, 36]. In the present study, when exposed the unanesthetized and unrestrained mice to high ambient temperature, overproduction of iNOS-dependent NO in their hypothalamus was also noted after the onset of heatstroke, which could be ameliorated by HBO therapy. It should be stressed that, in 

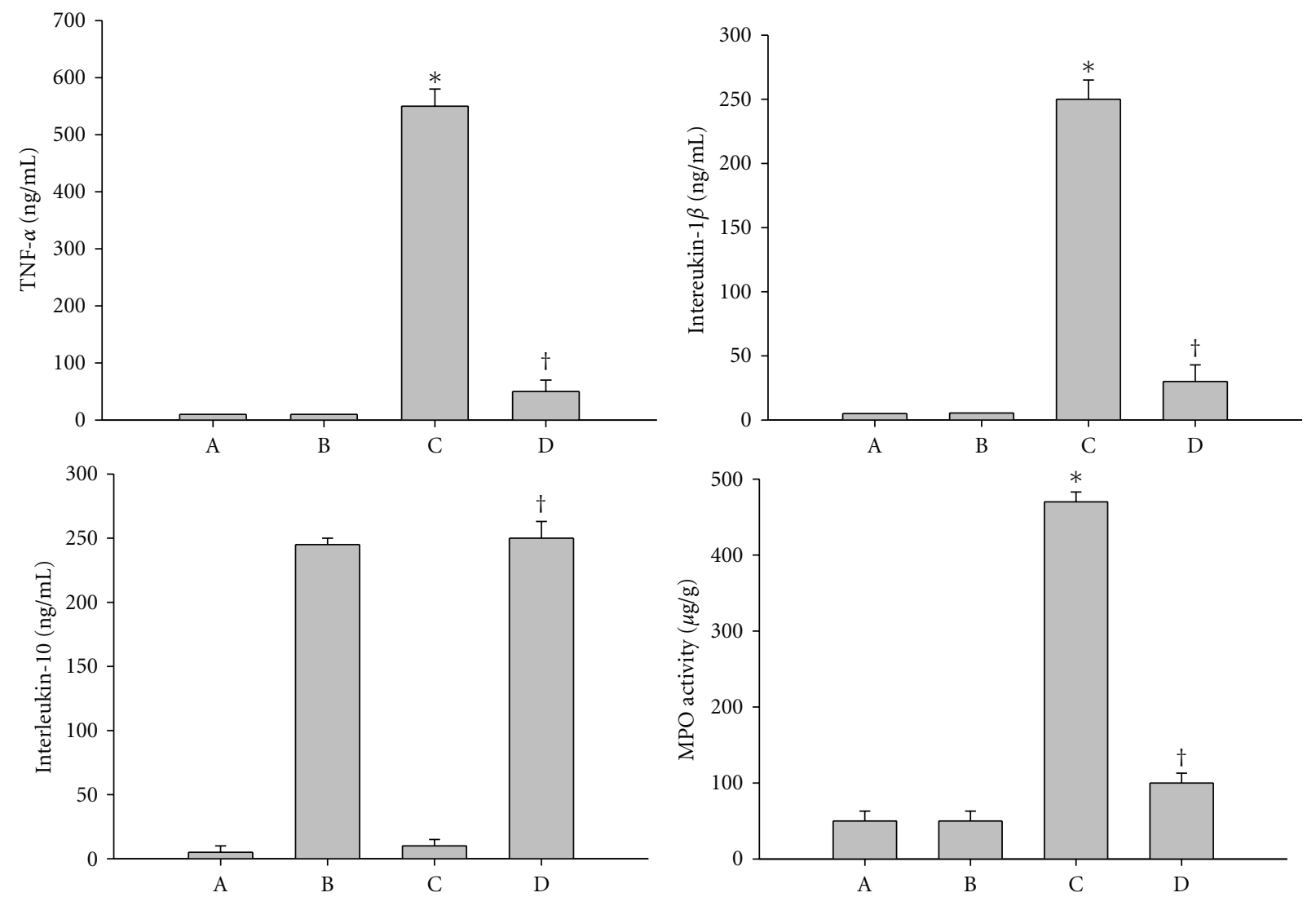

Figure 3: Effects of heat exposure $\left(42.4^{\circ} \mathrm{C}\right.$ for 1 hour) on hypothalamic levels of tumor necrosis factor- $\alpha$ (TNF- $\alpha$ ), interleukin- $1 \beta$, interleukin-10 and myeloperoxidase (MPO) of normothermic mice received normobaric air (A), normothermic mice received hyperbaric oxygen (B), heatstroke mice received normobaric air (C), and heatstroke mice received hyperbaric oxygen (D). ${ }^{*} P<.01$ compared with corresponding control values in group (A). ${ }^{\dagger} P<.01$ compared with corresponding control values in group $(\mathrm{C})$. Bars are mean $\pm \mathrm{SE}$ of eight mice per group. Samples were obtained at 4 hours after the termination of 1-hour heat exposure or the equivalent time for the normothermic controls.

TABLE 4: Mean ( \pm SE) number of iNOS-positive cells per hypothalamic section for different groups of mice $(n=8$ per group).

\begin{tabular}{lc}
\hline Treatment groups & iNOS-positive cells \\
\hline (1) Normothermic (NT) mice received normobaric oxygen (NBA) & 0 \\
(2) NT mice received hyperbaric oxygen (HBO) & 0 \\
(3) Heatstroke (HS) mice received NBA & $184 \pm 7^{*}$ \\
(4) HS mice received HBO & $35 \pm 3^{\dagger}$ \\
\hline
\end{tabular}

When exactly 4 hours post-HS, the iNOS staining were obtained.

* Compared with group 1.

${ }^{\dagger}$ Compared with group 3.

the current experiment, we measured only the hypothalamic levels of iNOS-dependent NOx. It is reasonable to assume that both iNOS-dependent and n-NOS-dependent NOx overproduction in different tissues following the onset of heatstroke can be ameliorated by HBO therapy.

In anesthetized rats, overproduction of both TNF- $\alpha$ and IL- $1 \beta$ in both the peripheral blood stream and the brain was noted during heatstroke $[37,38]$. The same phenomenon was also observed in the hypothalamus of unanesthetized, unrestrained mice attendant with heatstroke, as shown in the present results. HBO therapy greatly reduced the heat-induced overproduction of hypothalamic MPO, TNF$\alpha$ and IL- $1 \beta$, but enhanced the production of IL-10 in the hypothalamus. In fact, exogenous administration $f$ recombinant IL-10 was shown to protect mice from lethal endotoxemia by reducing TNF- $\alpha$ release [39]. In endotoxemic mice, neutralization of endogenous produced IL-10 resulted in an increased production of proinflammatory cytokines and an enhanced mortality [40]. IL-10 knockout mice had an increased extent of inflammatory illness [41] and higher mortality rates after experimental sepsis [39]. These observations imply that HBO may improve outcomes 

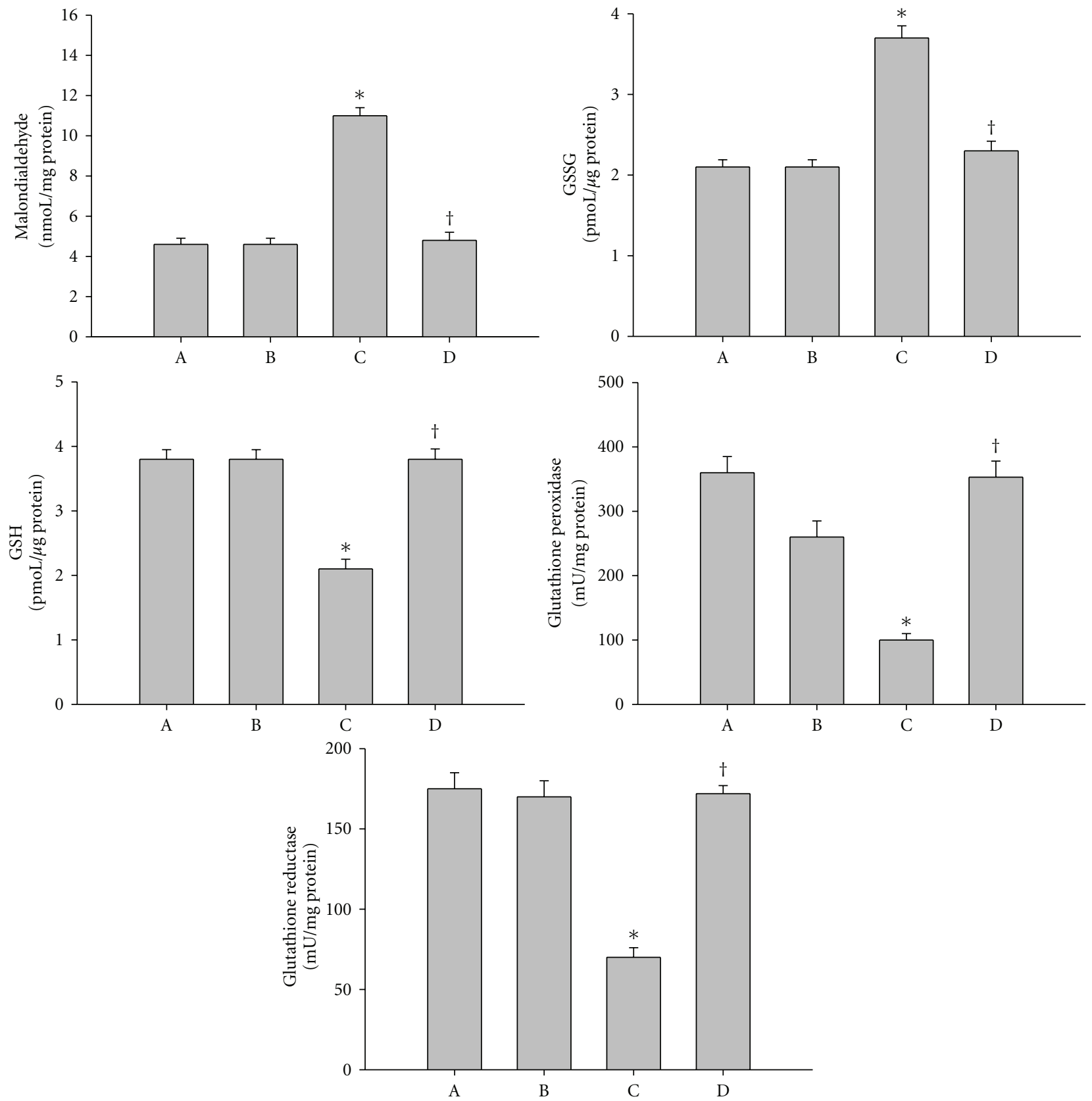

Figure 4: Effects of heat exposure $\left(42.4^{\circ} \mathrm{C}\right.$ for 1 hour) on hypothalamic levels of malondialdehyde, reduced form glutathione (GSH), oxidized form glutathione (GSSG), glutathione peroxidase, and glutathione reductase of normothermic mice received normobaric air (A) normothermic mice received hyperbaric oxygen (B) heatstroke mice received normobaric air (C), and heatstroke mice received hyperbaric oxygen (D). Bars are mean \pm SE of eight mice per group. ${ }^{*} P<.01$ compared with corresponding control values in group $(\mathrm{A}) .{ }^{\dagger} P<.01$ compared with corresponding values in group (C).

of heatstroke by increasing IL-10 but decreasing MPO, TNF$\alpha$ and IL- $1 \beta$ production in multiple organs (including the hypothalamus).

It has been previously demonstrated that, in anesthetized rats, the heatstroke-induced arterial hypotension, decreased arterial levels of $\mathrm{pH}, \mathrm{PaO}_{2}$, and $\mathrm{SO}_{2} \%$, and increased brain levels of pro-inflammatory cytokines, cellular ischemia and damage markers were all significantly reduced by $\mathrm{HBO}$ and, to some extent, by normobaric hyperoxia adopted immediately after the onset of heatstroke for 1 hour resuscitation [3]. In the current study, the heatstroke-induced ischemic and oxidative damage to the hypothalamus in unanesthetized mice could also be reduced by HBO therapy adopted 1 hour after termination of heat stress for 2 hours. Additionally, HBO therapy was used successfully treating a heatstroke patient with multiple organ dysfunction 


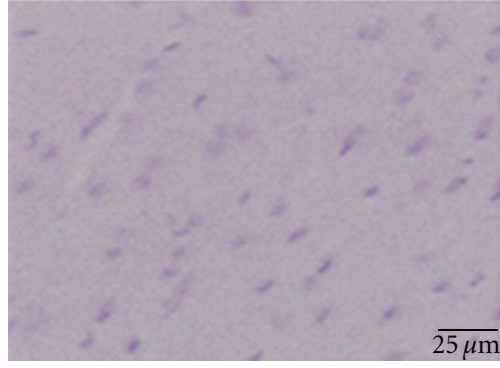

$\mathrm{NT}+\mathrm{NBA}$

(a)

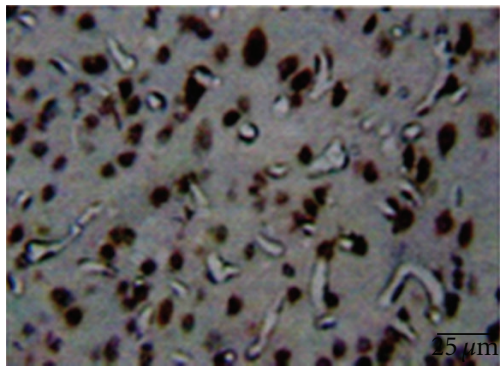

$\mathrm{HS}+\mathrm{NBA}$

(c)

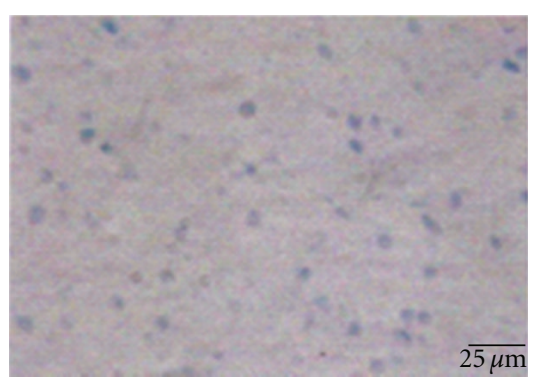

$\mathrm{NT}+\mathrm{HBO}$

(b)

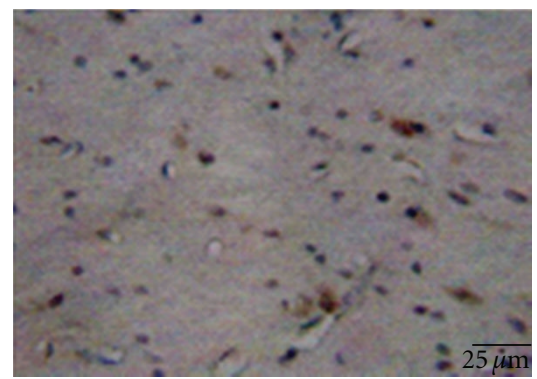

$\mathrm{HS}+\mathrm{HBO}$

(d)

FIGURE 5: Photomicrographs of inducible nitric oxide synthase (iNOS) staining of the hypothalamus of normothermic (NT) mouse received normobaric air (NBA) (NT+NBA), NT mouse received hyperbaric oxygen (HBO) (NT+HBO), heatstroke (HS) mouse received NBA (HS+NBA), or HS mouse received $\mathrm{HBO}(\mathrm{HS}+\mathrm{HBO})$. The animals were killed at 4 hours after the termination of 1-hour heat exposure or the equivalent time for the normothermic mouse.

[5]. Because the hypothalamus was believed to be the essential thermoregulatory center in brain [42], it is likely that thermoregulatory dysfunction could be resulted from hypothalamic ischemia and neuronal damage after the onset of heatstroke. Altering hypothalamic-pituitary-adrenal axis mechanisms would lead to multiorgan dysfunction or failure $[43,44]$. Thus, it appears that HBO may improve outcomes of heatstroke via normalization of the hypothalamic and thermoregulatory functions. Our data indicate that $\mathrm{HBO}$ is a promising strategy for treatment of heatstroke.

In the present study, the $\mathrm{HBO}$ was applied 1 hour after the induction of heatstroke for 2 hours. The time point was considered between 2-3 hours after the induction of heatstroke, when the mice were alive. If we considered a time point much later, this would help in translating the idea in human trials. In fact, hyperbaric air therapy or normobaric pure oxygen was also found to be beneficial in treating heatstroke in rats to some extents [3]. This may be easier to administer to patients in case it also helps in the recovery from heatstroke. We should further evaluate the basis of selection of the time of HBO therapy and duration of therapy in future studies.

Finally, it should be mentioned the heat shock preconditioning induced overproduction of heat shock protein (HSP) 72 and protected against heat-induced cerebral ischemia and damage [16, 17]. Again, future studies are required to ascertain whether HBO therapy improves outcomes of heatstroke through induction of HSP72 in multiple organs.

\section{Acknowledgments}

This work was supported by the National Science council of the Republic of China (Research Grant nos. NSC96-2314B-384-006-MY3 and NSC96-2314-B-384-003-MY3) and DOH99-TD-B-111-003, Center of Excellence for Clinical Trial and Research in Neuroscience.

\section{References}

[1] G. Leifer, "Hyperbaric oxygen therapy," American Journal of Nursing, vol. 101, no. 8, pp. 26-34, 2001.

[2] K.-C. Niu, M.-T. Lin, and C.-P. Chang, "Hyperbaric oxygen improves survival in heatstroke rats by reducing multiorgan dysfunction and brain oxidative stress," European Journal of Pharmacology, vol. 569, no. 1-2, pp. 94-102, 2007.

[3] H.-M. Tsai, C.-J. Gao, W.-X. Li, M.-T. Lin, and K.-C. Niu, "Resuscitation from experimental heatstroke by hyperbaric oxygen therapy," Critical Care Medicine, vol. 33, no. 4, pp. 813818, 2005.

[4] C.-H. Yeh, Z.-C. Chen, C.-C. Hsu, M.-T. Lin, and C.C. Chen, "Protection in rats with heatstroke: hyperbaric oxygen vs activated protein C therapy," European Journal of Pharmacology, vol. 635, no. 1-3, pp. 103-108, 2010.

[5] K.-C. Niu, C.-K. Chang, M.-T. Lin, and K.-F. Huang, "A hyperbaric oxygen therapy approach to heat stroke with multiple organ dysfunction: a case report," Chinese Journal of Physiology, vol. 52, no. 3, pp. 169-172, 2009. 
[6] Y. T. Chou, S. T. Lai, C. C. Lee, and M. T. Lin, "Hypothermia attenuates circulatory shock and cerebral ischemia in experimental heatstroke," Shock, vol. 19, no. 4, pp. 388-393, 2003.

[7] K.-H. Shen, C.-H. Lin, H.-K. Chang, W.-C. Chen, and S.-H. Chen, "Premarin can act via estrogen receptors to rescue mice from heatstroke-induced lethality," Shock, vol. 30, no. 6, pp. 668-674, 2008.

[8] Z.-C. Chen, W.-S. Wu, M.-T. Lin, and C.-C. Hsu, "Protective effect of transgenic expression of porcine heat shock protein 70 on hypothalamic ischemic and oxidative damage in a mouse model of heatstroke," BMC Neuroscience, vol. 10, no. 9, pp. 111-119, 2009.

[9] S. Chatterjee, S. Premachandran, D. Sharma, R. S. Bagewadikar, and T. B. Poduval, "Therapeutic treatment with L-arginine rescues mice from heat stroke-induced death: physiological and molecular mechanisms," Shock, vol. 24, no. 4, pp. 341-347, 2005.

[10] S. Chatterjee, S. Premachandran, R. S. Bagewadikar, S. Bhattacharya, S. Chattopadhyay, and T. B. Poduval, "Arginine metabolic pathways determine its therapeutic benefit in experimental heatstroke: role of Th1/Th2 cytokine balance," Nitric Oxide, vol. 15, no. 4, pp. 408-416, 2006.

[11] J. Stitt, "Central regulation of body temperature," in Perspectives in Exercise Science and Sports Medicine, C. V. Gisolfi, D. R. Lamb, and E. R. Nadel, Eds., vol. 6, pp. 2-39, Cooper, Carmel, Ind, USA, 1993.

[12] K. Matsukawa, "Central control of the cardiovascular system during exercise," in Exercise, Nutrition, and Environmental Stress, H. Nose, C. V. Gisolfi, and K. Imaizumi, Eds., vol. 1, pp. 39-64, Coopers, Traverse City, Mich, USA, 2001.

[13] S. Hideaki, "Stress response to exercise and its possible hypothalamic regulation: role of arginine-vasopressin," in Exercise, Nutrition, and Environmental Stress, H. Nose, C. V. Gisolfi, and K. Imaizumi, Eds., vol. 1, pp. 21-38, Coopers, Transverse City, Mich, USA, 2001.

[14] W. A. Pulsinelli, J. B. Brierley, and F. Plum, "Temporal profile of neuronal damage in a model of transient forebrain ischemia," Annals of Neurology, vol. 11, no. 5, pp. 491-498, 1982.

[15] H. Togashi, K. Mori, K.-I. Ueno et al., "Consecutive evaluation of nitric oxide production after transient cerebral ischemia in the rat hippocampus using in vivo brain microdialysis," Neuroscience Letters, vol. 240, no. 1, pp. 53-57, 1998.

[16] C.-Y. Yang and M.-T. Lin, "Oxidative stress in rats with heatstroke-induced cerebral ischemia," Stroke, vol. 33, no. 3, pp. 790-794, 2002.

[17] J.-L. Wang, D.-S. Ke, and M.-T. Lin, "Heat shock pretreatment may protect against heatstroke-induced circulatory shock and cerebral ischemia by reducing oxidative stress and energy depletion," Shock, vol. 23, no. 2, pp. 161-167, 2005.

[18] F. Tietze, "Enzymic method for quantitative determination of nanogram amounts of total and oxidized glutathione: applications to mammalian blood and other tissues," Analytical Biochemistry, vol. 27, no. 3, pp. 502-522, 1969.

[19] O. W. Griffith, "Determination of glutathione and glutathione disulfide using glutathione reductase and 2-vinylpyridine," Analytical Biochemistry, vol. 106, no. 1, pp. 207-212, 1980.

[20] K. M. Mullane, R. Kraemer, and B. Smith, "Myeloperoxidase activity as a quantitative assessment of neutrophil infiltration into ischemic myocardium," Journal of Pharmacological Methods, vol. 14, no. 3, pp. 157-167, 1985.
[21] L. Hillered and L. Persson, "Neurochemical monitoring of the acutely injured human brain," Scandinavian Journal of Clinical and Laboratory Investigation, Supplement, vol. 59, no. 229, pp. 9-18, 1999.

[22] L. Hillered, J. Valtysson, P. Enblad, and L. Persson, "Interstitial glycerol as a marker for membrane phospholipid degradation in the acutely injured human brain," Journal of Neurology, Neurosurgery and Psychiatry, vol. 64, no. 4, pp. 486-491, 1998.

[23] L. Hillered, L. Persson, U. Ponten, and U. Ungerstedt, "Neurometabolic monitoring of the ischaemic human brain using microdialysis," Acta Neurochirurgica, vol. 102, no. 3-4, pp. 91-97, 1990.

[24] U. Ungerstedt, "Microdialysis-a new technique for monitoring local tissue events in the clinic," Acta Anaesthesiologica Scandinavica. Supplementum, vol. 110, p. 123, 1997.

[25] O. G. Nilsson, H. Säveland, F. Boris-Möller, L. Brandt, and T. Wieloch, "Increased levels of glutamate in patients with subarachnoid haemorrhage as measured by intracerebral microdialysis," Acta Neurochirurgica, Supplement, vol. 67, pp. 45-47, 1996.

[26] L. Persson and L. Hillered, "Chemical monitoring of neurosurgical intensive care patients using intracerebral microdialysis," Journal of Neurosurgery, vol. 76, no. 1, pp. 72-80, 1992.

[27] C. Iadecola, "Bright and dark sides of nitric oxide in ischemic brain injury," Trends in Neurosciences, vol. 20, no. 3, pp. 132139, 1997.

[28] C. F. Chang, K. C. Niu, B. J. Hoffer, Y. Wang, and C. V. Borlongan, "Hyperbaric oxygen therapy for treatment of postischemic stroke in adult rats," Experimental Neurology, vol. 166, no. 2, pp. 298-306, 2000.

[29] L. H. Fenton and M. B. Robinson, "Repeated exposure to hyperbaric oxygen sensitizes rats to oxygen-induced seizures," Brain Research, vol. 632, no. 1-2, pp. 143-149, 1993.

[30] C. F. Zwemer, E. M. O'Connor, S. E. Whitesall, and L. G. D'Alecy, "Gender differences in 24-hour outcome following resuscitation after 9 minutes of cardiac arrest in dogs," Critical Care Medicine, vol. 25, no. 2, pp. 330-338, 1997.

[31] H. S. Mickel, Y. N. Vaishnav, O. Kempski, et al., "Breathing $100 \%$ oxygen after global brain ischemia in Mongolian gerbils results in increased lipid peroxidation and increased mortality," Stroke, vol. 18, no. 2, pp. 426-430, 1987.

[32] C.-K. Chang, C.-P. Chang, S.-Y. Liu, and M.-T. Lin, "Oxidative stress and ischemic injuries in heat stroke," Progress in Brain Research, vol. 162, pp. 525-546, 2007.

[33] C.-P. Chang, C.-C. Lee, S.-H. Chen, and M.-T. Lin, "Aminoguanidine protects against intracranial hypertension and cerebral ischemic injury in experimental heatstroke," Journal of Pharmacological Sciences, vol. 95, no. 1, pp. 56-64, 2004.

[34] D. M. Hall, G. R. Buettner, L. W. Oberley, L. Xu, R. D. Matthes, and C. V. Gisolfi, "Mechanisms of circulatory and intestinal barrier dysfunction during whole body hyperthermia," American Journal of Physiology, vol. 280, no. 2, pp. H509-H521, 2001.

[35] H. S. Sharma, J. Westman, P. Alm, P.-O. Sjöquist, J. CervósNavarro, and F. Nyberg, "Involvement of nitric oxide in the pathophysiology of acute heat stress in the rat," Annals of the New York Academy of Sciences, vol. 813, pp. 581-590, 1997.

[36] B.-C. Cheng, C.-P. Chang, M.-T. Lin, and C.-C. Lee, "Inhibition of neuronal nitric oxide synthase causes attenuation of cerebrovascular dysfunction in experimental heatstroke," Neuropharmacology, vol. 52, no. 2, pp. 297-305, 2007.

[37] M. T. Lin, H. H. Liu, and Y. L. Yang, "Involvement of interleukin-1 receptor mechanisms in development of arterial 
hypotension in rat heatstroke," American Journal of Physiology, vol. 273, no. 4, part 2, pp. H2072-H2077, 1997.

[38] C.-C. Liu, C.-H. Chien, and M.-T. Lin, "Glucocorticoids reduce interleukin- $1 \beta$ concentration and result in neuroprotective effects in rat heatstroke," Journal of Physiology, vol. 527, no. 2, pp. 333-343, 2000.

[39] C. Gerard, C. Bruyns, A. Marchant et al., "Interleukin 10 reduces the release of tumor necrosis factor and prevents lethality in experimental endotoxemia," Journal of Experimental Medicine, vol. 177, no. 2, pp. 547-550, 1993.

[40] K. W. Moore, R. De Waal Malefyt, R. L. Coffman, and A. O'Garra, "Interleukin-10 and the interleukin-10 receptor," Annual Review of Immunology, vol. 19, pp. 683-765, 2001.

[41] D. M. Rennick, M. M. Fort, and N. J. Davidson, "Studies with IL-10 ${ }^{-/-}$mice: an overview," Journal of Leukocyte Biology, vol. 61, no. 4, pp. 389-396, 1997.

[42] G. Wright, E. Knecht, and D. Wasserman, "Colonic heating patterns and the variation of thermal resistance among rats," Journal of Applied Physiology, vol. 43, no. 1, pp. 59-64, 1977.

[43] K. Matsukawa, "Central control of the cardiovascular system during exercise," in Exercise, Nutrition, and Environmental Stress, H. Nose, C. V. Gisolfi, and K. Imaizumi, Eds., vol. 1, pp. 39-64, Coopers, Transverse City, Mich, USA, 2001.

[44] S. Hideaki, "Stress response to exercise and its possible hypothalamic regulation: role of arginine-vasopressin," in Exercise, Nutrition, and Environmental Stress, H. Nose, C. V. Gisolfi, and K. Imaizumi, Eds., vol. 1, pp. 21-38, Coopers, Transverse City, Mich, USA, 2001. 


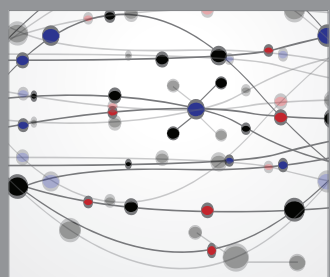

The Scientific World Journal
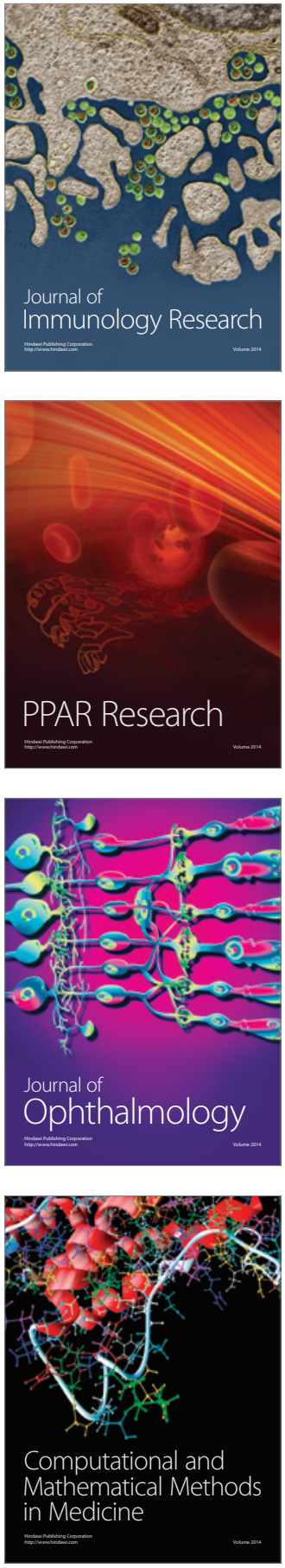

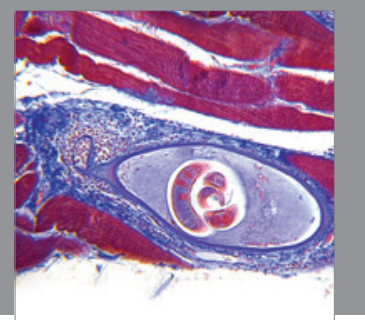

Gastroenterology

Research and Practice
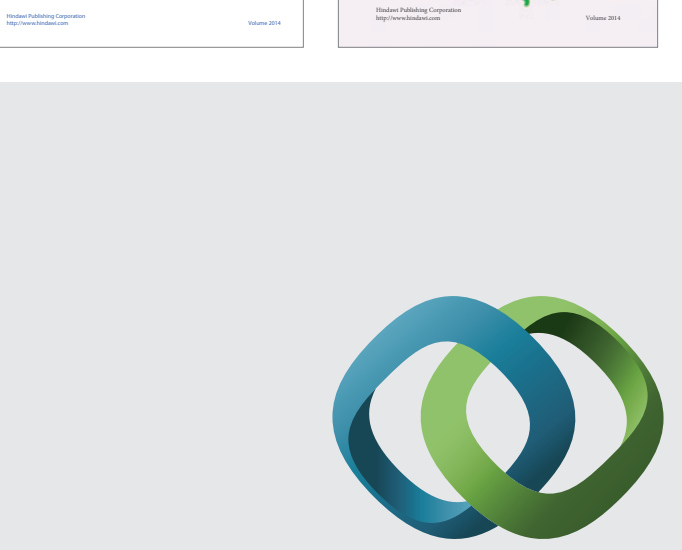

\section{Hindawi}

Submit your manuscripts at

http://www.hindawi.com
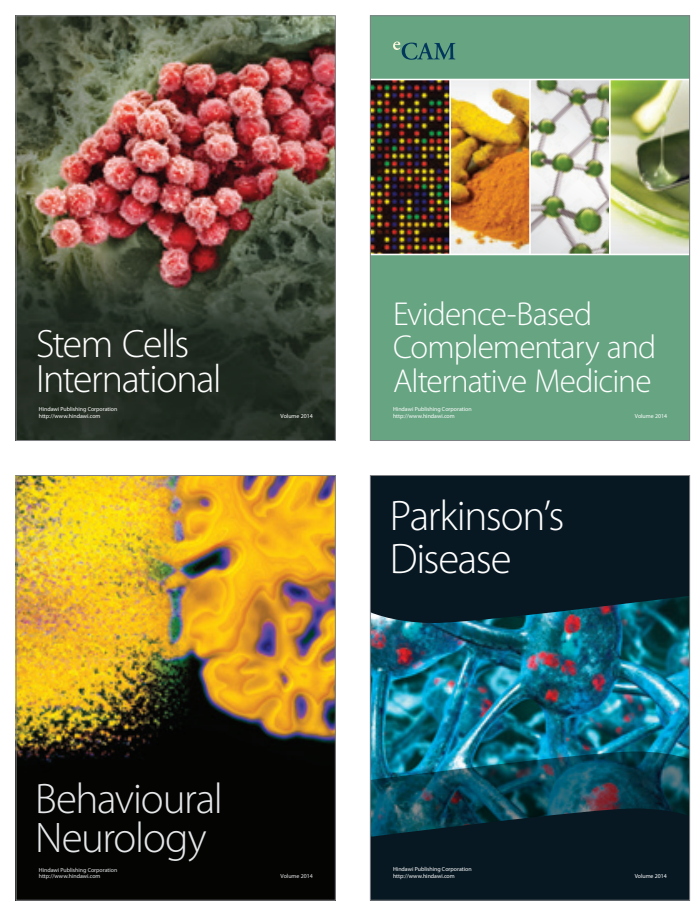

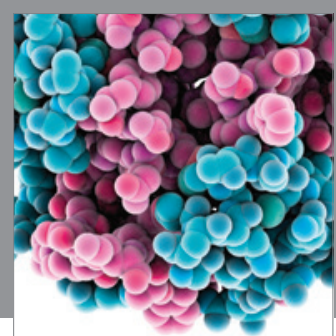

Journal of
Diabetes Research

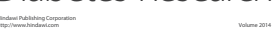

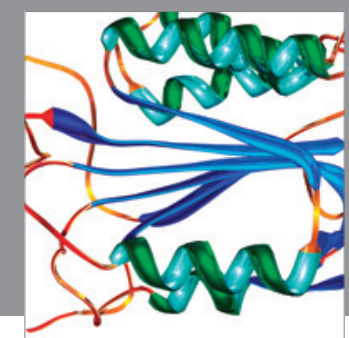

Disease Markers
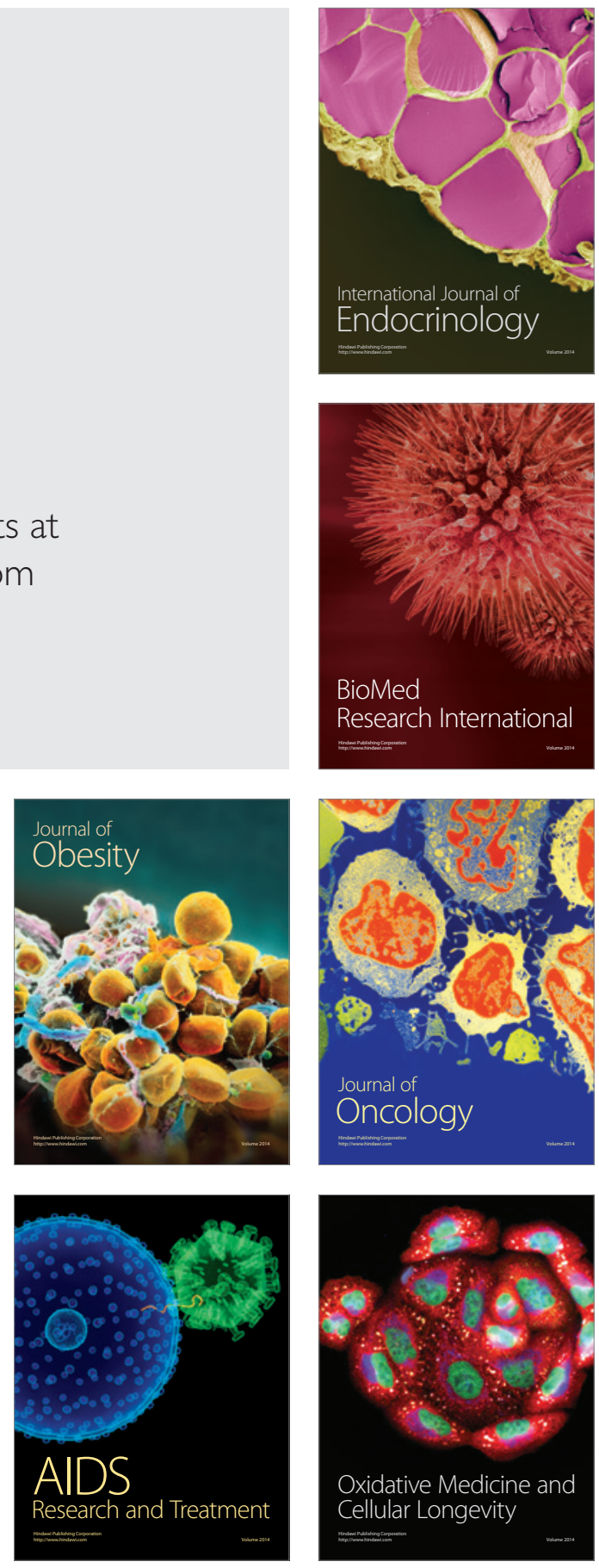